\title{
Täterinnen - die Gewaltausübung von Frauen im privaten Raum im Kontext der feministischen Diskussion über Gewalt im Geschlechterverhältnis \\ Barbara Kavemann
}

Seit etwa 2002 wird eine (fach)öffentliche Auseinandersetzung mit der Gewaltausübung von Frauen in Paarbeziehungen geführt - mal mehr, mal weniger intensiv und teilweise deutlich von ideologischen Positionen zulasten empirischer Fakten dominiert. Eine Seite der Kontroverse will bei Frauen die gleiche Gewaltbereitschaft und Gewalttätigkeit sehen wie bei Männern und zieht dafür Studien zum Beweis heran. Die bislang geführte Diskussion über Gewalt gegen Frauen wird als Vernebelung der tatsächlichen Verhältnisse kritisiert, die das Leid von Männern ignoriere und Frauen durch rechtliche Reformen und frauenspezifische Unterstützungsangebote in die Lage versetze, diese in Beziehungskonflikten gegen Männer zu instrumentalisieren. Es sei eine gesellschaftliche Ungerechtigkeit und eine Verschwendung von Steuergeldern, Schutzund Beratungseinrichtungen für Frauen zu fördern. Die entgegen gesetzte Position wirft den Vertreter/innen dieser Argumentation ebenfalls eine ignorante Haltung vor und sieht hierin eine antifeministische Bewegung bzw. eine Väterrechtsbewegung, die die Errungenschaften der Frauenbewegung gefährde und das Rad der Geschichte zurückdrehen wolle und auch sie berufen sich auf Forschungsergebnisse. Das Verharren auf diesen Positionen ist nicht produktiv, denn sie verfestigt den Status quo und verhindert, dass wir über das Gewalterleiden von Männern oder die Gewalttätigkeit von Frauen Neues lernen.

\section{Gibt es einen Weg aus der Sackgasse?}

Um aus Sackgassen in der Diskussion herauszukommen, hilft es oft, einen Blickwechsel vorzunehmen, eine neue Perspektive auszuprobieren. Hilfreich war in diesem Sinne die Einbindung der Diskussion über Gewalt im den Geschlechterverhältnis in den Menschenrechtsdiskurs. Geschlechtsspezifische Diskriminierungen, Gewalt im privaten Raum - vor allem in intimen Beziehungen wurde lange nicht als Menschenrechtsverletzungen definiert. Dies hat sich u.a. mit dem „Internationalen Übereinkommen gegen jede Form der Diskriminierung der Frau“ von 1979 (CEDAW), ferner als Ergebnis der Wiener Menschenrechtskonferenz von
1993 geändert. Die Weltfrauenkonferenzen machten das Problem der Gewalt in Ehe und Beziehung weltweit zum Thema von Menschenrechtsverletzungen.

Sobald die Gewalt in intimen Partnerschaften in diesem Rahmen diskutiert wurde, konnte vom Konzept der „unschuldiger“ Opfer zur einer kritischen Parteilichkeit (Kavemann 1997) übergegangen werden, die die Geschlechtsspezifik der Gewalt klar erkannte, aber das Problem nicht durch Vereinfachungen zu lösen versuchte. Soziale Gruppen wie z.B. Männer und Frauen, Mehrheiten und Minderheiten treten sich als ,verletzungsoffen“ bzw. als „verletzungsmächtig“ gegenüber, Männer und Frauen können je nach Kontext sowohl zu den Verletzungsoffenen wie auch zu den Verletzungsmächtigen zählen, bzw. - und das ist die spezifische Schwierigkeit - beides zugleich darstellen. Frauen und Mädchen als gesellschaftliche Gruppe gestalten mit eigenen Interessen die Gesellschaft mit und tragen zum weiteren Bestand patriarchaler Strukturen bei, auch wenn sie gleichzeitig für den Abbau von Gewalt und für Gleichberechtigung kämpfen (ThürmerRohr 1989). Frauen und Mädchen sind aktiver Teil der Welt, in der sie leben. Die Eingebundenheit der Frauen in patriarchale Logik und Aktion zu sehen, führte zu einem neuen Verständnis von der Eingebundenheit der Gewaltopfer in die Gewaltsituation und zu einer intensiven Auseinandersetzung mit der Begrifflichkeit vom Opfer jenseits einer Opferdiffamierung oder einer Opferidealisierung (Kavemann 1995 und 1998).

Heute ist es nicht mehr ungewöhnlich, dass auch in feministischen bzw. frauenspezifischen Einrichtungen Gewalt von Mädchen und Frauen Thema der Beratungs- und Unterstützungsarbeit ist. Hier ist viel in Bewegung. Als ich vor einigen Jahren auf einer Fachtagung Pädagoginnen aus der Mädchenarbeit zuhörte, die ihre Konzepte der Arbeit mit gewaltbereiten Mädchen diskutierten, war ich beeindruck. Eines jedoch gab mir zu denken: Auf meine Frage, was denn die Mädchen an der Gewalt fasziniere, gab es wenig Antworten. Die Berührung mit der Gewalt, das Interesse am Phänomen Gewalt und dem Reiz, der von Gewalt ausgeht, blieb vorsichtig und reduziert. Die Entwicklung ist weitergegangen. Die Tagung zu Täterinnen, die im Frühjahr 2009 an der Universität Osnabrück durchgeführt wurde, zeigte, dass es, was die Gewalttätigkeit von Mädchen betrifft, neue und interessante Forschung gibt, die gerade den Kick und das Ziel der Gewalt von Mädchen hinterfragt (Bruhns 2006, Silkenbeumer 2007, Artz 1998).

Die anhaltenden und in den letzten Jahren eskalierenden Angriffe auf feministische Arbeit gegen sexualisierte Gewalt und Gewalt in Paarbeziehungen drängten den feministischen Diskurs stark in die Defensive. Starke Tendenzen in der öffentlichen und fachöffentlichen Diskussion warfen engagierten Frauen vor, Fakten übertrieben oder verdreht und die Gewaltausübung von Frauen verschwiegen zu haben. Aus der Defensive heraus war es schwierig, sich der Problematik der Gewalt von Frauen unvoreingenommen anzunähern. Verstärkt wurde der Konflikt durch Wissenschaftler und Aktivisten der Männerbewegung, die begannen, auf die Gewalt von Frauen in Partnerschaften aufmerksam zu machen. Sie bezogen sich dabei auf eine Fülle vorliegender Studien (Archer 2000), aus denen hervorgeht, dass Frauen in gleicher Häufigkeit wie Männer Gewalt gegen Partner ausüben. Viele Frauen zweifelten diese Ergebnisse rundweg an, so wenig passten sie zu den Erfahrungen, die sie im Laufe vieler Jahre in Schutz und Unterstützungseinrichtungen für von Gewalt betroffene Frauen gesammelt hatten.

Die Diskussion über die Geschlechterverteilung bei Gewalt in intimem Beziehungen leidet unter einer Uneindeutigkeit der Begriffe. Die Begriffe „Gewalt gegen Frauen“ und „Gewalt gegen Männer" erfassen unterschiedliche Spektren von Gewalt. Ein Blick in die beiden in 2004 in Deutschland vorgelegten Studien zu Gewalt gegen Frauen (Schröttle u.a. 2004) und Gewalt gegen Männer (Forschungsverbund Gewalt gegen Männer 2004) - zeigt Bedarf an der Klärung von Begrifflichkeiten. Gleichzeitig wird deutlich, wie die historisch entstandenen Begriffe die Reichweite und Schwerpunktsetzung der Diskussion geprägt haben. 
Zuerst fällt die Unterschiedlichkeit der Begriffe „Gewalt gegen Frauen“ und „Gewalt gegen Männer“ auf. Beide Begriffe umfassen nicht das gleiche Spektrum an Gewaltverhältnissen und weisen auf einen Bedarf an unterschiedlichen Interventions- und Präventionsansätzen hin. "Gewalt gegen Frauen" umfasst zwar vielfältige Ausprägungen von Gewalterleben, jedoch ausschließlich Gewalt von Männern gegen Frauen und bedeutet eine Kritik der Konstruktion des Geschlechterverhältnisses. Gewalt in der Arbeitswelt wird in der Form sexueller Belästigung am Arbeitsplatz einbezogen, Gewalt in Kriegen und bewaffneten Konflikten fast ausschließlich in Form von Vergewaltigungen. Gewalt in der Kindheit wird, wenn überhaupt, dann nur in Form des sexuellen Missbrauchs mit erfasst. Kindesmisshandlung und Vernachlässigung, institutionelle Gewalt im Alter oder Gewalt durch Partnerinnen oder andere Frauen ist in aller Regel nicht gemeint, wenn von „Gewalt gegen Frauen“ gesprochen wird. Die Auseinandersetzung mit Gewalt zwischen Frauen und Gewalt in lesbischen Beziehungen ist eine wenig beachtete Diskussion geblieben. Frauen sprechen über das andere Geschlecht, wenn sie von Gewalt sprechen. Die Anklage, die dem Begriff „Gewalt gegen Frauen“ inhärent ist, richtet sich gegen gesellschaftliche Strukturen der Benachteiligung und Ausgrenzung entlang der Geschlechtergrenzen.

"Gewalt gegen Männer" - wie der Begriff in der Pilotstudie und auch in der breiten Diskussion verwendet wird - umfasst im Unterschied dazu alle Gewalt im Leben von Männern, unabhängig vom Alter der Gewaltbetroffenen und von der Person der Gewalthandelnden. Es geht darum, zu etablieren, dass auch Männer vulnerabel sind. Sie sollen nicht nur als Täter sondern auch als Opfer sichtbar werden (vgl. Lenz 2000). Der Begriff ist zu verstehen als eine Kritik dominanter Männlichkeitskonstrukte. Gewalt in der Kindheit ist in all ihrer Vielfalt darin enthalten, Gewalt im Arbeitsleben - vor allem in der Form psychischer Gewalt - nimmt einen zentralen Platz ein. Gewalt gegen Männer wird überwiegend von Männern verübt. Die Anklage richtet sich somit nicht gegen Frauen - außer in den konkreten Fällen, in denen Frauen gewalttätig gegen Jungen und Männer sind - sondern gegen eine Gesellschaft, die Männern die Anpassung an schädigende Männlichkeitskonzepte abverlangt. Männer müssen vor allem über das eigene Geschlecht sprechen, wenn sie von Gewalt sprechen. Eine Thematisierung der Ambivalenz zwischen Leiden an den gesellschaftlichen Verhältnisse und dem Genuss von Privilegien oder der Gleichzeitigkeit von Täter- und Opfersein hat erst begonnen, hat aber das Potential, maßgeblich zu gesellschaftlichem Wandel beizutragen. Sie wird teilweise von angestrengtem Bemühen überlagert, den Nachweis zu erbringen, dass Frauen ebenso gewalttätig seien wie Männer.

Die Klärung, welches Spektrum von Gewalt die jeweils benutzen Begriffe umfassen, ist von Bedeutung für Intervention und Prävention. Wenn Gewalterleben und Gewalthandeln nicht präzisiert werden, bleibt unscharf, was unter Gewalt im engeren und im weiteren Sinne zu fassen ist. Wird die subjektive Bewertung von Erlebnissen und Taten nicht präzisiert, wird nur wenig Erkenntnis über die Bedeutung von Gewalt für Frauen und Männer gewonnen. Die Bedeutung, die Gewalttätige und die Gewalt erleidende der Gewalt zumessen ist aber der Schlüssel für wirksame Intervention und bedarfsgerechten Schutz.

\section{Was sagt die Forschung?}

Kritische Wissenschaftlerinnen und Wissenschaftler setzten sich seit Jahren mit vorliegenden Forschungsergebnisse auseinander und begannen eine weiterführende Diskussion (Kavemann 2003, Gloor/Meier 2003, Johnson 1995 und 2005, Kimmel 2002). Die internationale Fachzeitschrift „Violence Against Women “ widmete 2002 dem Thema der Gewalt durch Frauen zwei Sonderhefte. Wird die vorliegende Forschung unvoreingenommen betrachtet, zeigt sich ein Bild, das spezifische Gemeinsamkeiten und
Unterschiede aufweist. Frauen und Männer sind im Laufe ihres Lebens häufig Opfer von Gewalt - Männer etwas häufiger als Frauen - und in beiden Fällen sind die Gewalttäter überwiegend Männer. Unterschiedlich ist die Art der Gewalt, der beide Geschlechter ausgesetzt sind: Soweit bislang bekannt, sind Männer häufiger als Frauen Opfer von Körperverletzung, Frauen hingegen deutlich häufiger als Männer Opfer von Vergewaltigung und anderen Formen sexualisierter Gewalt.

Der Kontext des Gewalterlebens unterscheidet sich ebenfalls nach Geschlecht: Frauen werden häufiger Opfer durch Beziehungspartner oder Familienangehörige, Männer häufiger durch Bekannte oder Fremde. Frauen erleiden mehr Gewalt im privaten Raum, Männer häufiger im öffentlichen Raum.

Auch die Risiken, die mit der Gewalt einhergehen, sind unterschiedlich: Frauen werden häufiger als Männer im Kontext häuslicher Gewalt verletzt oder getötet. Das Verletzungsrisiko für Frauen steigt, wenn die körperliche bzw. sexuelle Gewalt von einem Beziehungspartner ausgeht. Für Männer sinkt das Verletzungsrisiko, wenn die Gewalt von ihrer Beziehungspartnerin ausgeht (Schröttle u.a.:2004, Tjaden \& Thoennes:2000, National Crime Victimization Study:1994).

Die Gewaltausübung von Frauen gegen Männer, mit denen sie zusammenleben, ist empirisch belegt: Sie ist verhältnismäßig häufig, sie verursacht verhältnismäßig selten Verletzungen und löst auch verhältnismäßig selten Angst aus.

Abbildung 1: Geschlechterverteilung bei sexueller und körperlicher Gewalt in \% (Thaden und Thoennes 2000)

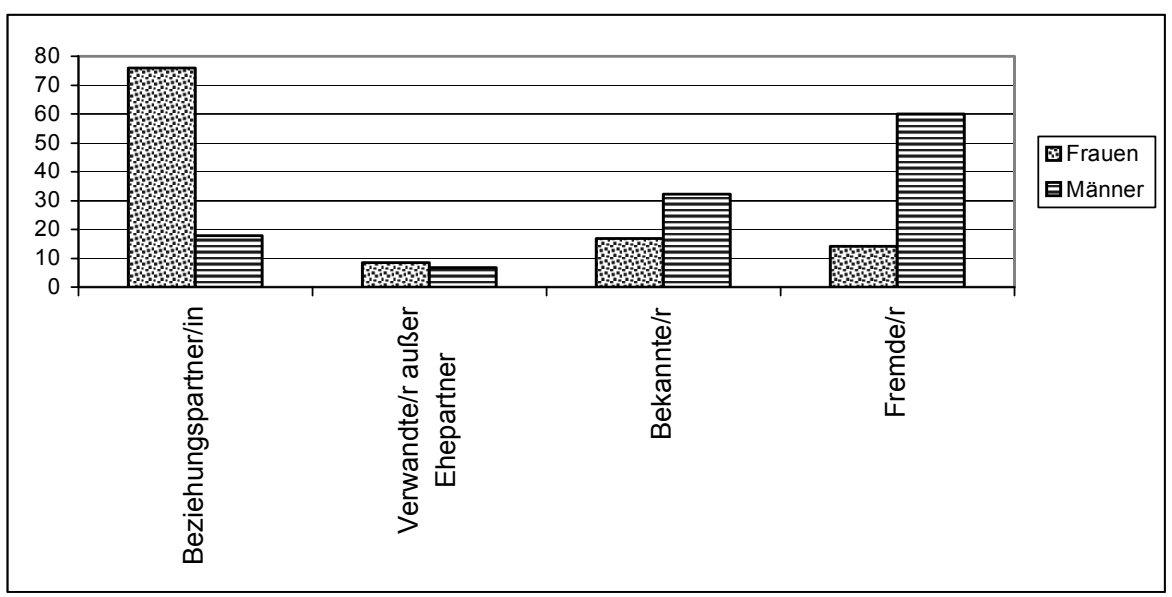


Abbildung 2: Bedrohung und Angst bei männlichen und weiblichen Opfern von Gewalt, die von einem Ehepartner / einer Ehepartnerin ausging (Thaden und Thoennes 2000)

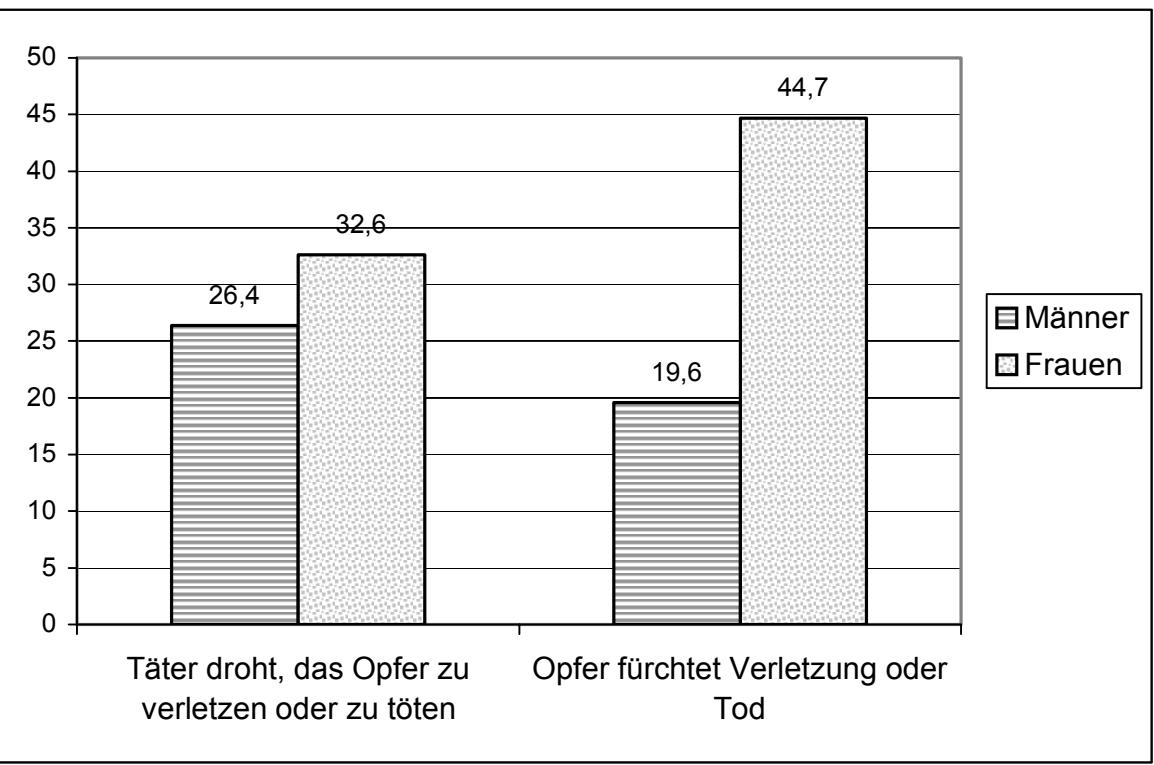

Damit hatte die Diskussion über Gewalt im Geschlechterverhältnis einen großen Schritt nach vorne getan. Zunehmend wurde thematisiert, dass Gewalt nicht gleich Gewalt ist, dass sowohl der Kontext als auch die Folgen von Gewalthandlungen in der Forschung berücksichtigt werden müssen. Dies ermöglichte eine differenzierte Betrachtung der Gewalt, die von Frauen und Männern ausgeht, aber auch eine bislang nicht selbstverständliche Differenzierung der Gewaltverhältnisse, in denen Frauen leben.

Die Veränderung setzte auf zwei Ebenen an:

(1) Nachdem es lange Zeit im Sinne der Durchsetzung des Themas in der Öffentlichkeit und auf der politischen Agenda als notwendig erachtet worden war, die schwere, eskalierende und lebensbedrohliche Gewalt in den Vordergrund zu rücken, wurde nun die Unterschiedlichkeit der Gewalt, die Frauen erleiden, auf neue Art und Weise diskutiert. Darüber hinaus begann die Forschung damit, unterschiedliche Muster von Gewaltverhältnissen und daraus resultierenden differenzierten Unterstützungsbedarf zu entwickeln (Johnson 1995, Piispa 2002, Helfferich 2004, Schröttle 2004).

(2) Bislang herrschte in der öffentlichen Diskussion häuslicher Gewalt das Bild der eskalierenden Gewaltspirale vor, die die Handlungsfähigkeit der Betroffenen reduzierte und die Risiken potenzierte. Es zeigte sich, dass ein einzelnes Modell der Beschreibung von Gewalthandlungen und Gewaltverhältnissen zu kurz greift. Wenn nur eskalierende, chronifizierte und/oder lebensbedrohliche Gewalt in den Blick genommen wird, wird die Gewalt von Frauen in Paarbeziehungen kaum sichtbar denn sie ist eher selten von dieser Intensität.

Hilfreich zum Verständnis der Unterschiede war die differenzieren de Analyse vorliegender Forschung durch Michael Johnson. Er unterscheidet vor allem zwei Formen von Gewaltverhältnissen:

- „intimate terrorism“ (Johnson 1995) oder auch „Gewalt als systematisches Kontrollverhalten“ (Gloor/Meier 2003)

Diese Gewalt dient der Ausübung von Kontrolle und Beherrschung in der Partnerschaft. Hier besteht eine starke Verknüpfung mit frauenfeindlichen Einstellungen der Täter - nicht nur Feindseligkeit gegenüber der Partnerin, sondern gegenüber Frauen im allgemeinen. Häufig ist hier die eskalierende Gewaltspirale anzutreffen, Gewalt und Kontrolle engen den Handlungsspielraum der Partnerin ein und machen ein Entkommen aus der Situation schwer. Dieses Muster ist stark ausgeprägte geschlechtsspezifische Gewalt, es wird ganz überwiegend von männlichen Tätern ausgeübt.

Dieser Form der Gewalttätigkeit steht eine andere gegenüber:

- „situational couple violence“ (Johnson 1995) oder auch „Gewalt als spontanes Konfliktverhalten“ (Gloor/Meier 2003)

Diese Gewalt ist nicht eingebettet in ein Muster von Macht und Kontrolle. Es han- delt sich um Gewalthandlungen in einzelnen eskalierten Konflikten oder in Serien von Konflikten. Meist führen sie nicht zu Verletzungen. In der Regel ist keine Eskalation nach dem Modell der Gewaltspirale zu beobachten. Bei dieser Form ist eine Gleichverteilung nach Geschlecht (ca. 50\% männliche und $50 \%$ weibliche Täter/innen) anzutreffen (ebd.).

Johnsons Differenzierungen lassen auch die Diskrepanzen zwischen den Forschungsergebnissen verstehbar werden. Geht es um häusliche Gewalt - um Gewaltausübung in intimen Beziehungen zwischen Erwachsenen - dann ist festzustellen, dass vorliegende Studien unterschiedliche Phänomene messen: Während in Studien, die einen hohen Anteil männlicher Opfer und weiblicher Täterinnen aufweisen (vgl. Archer 2000), überwiegend „common couple violence“ erhoben wird, geht es in Studien, die einen überwiegenden Anteil weiblicher Opfer und männlicher Täter nachweisen, um systematische Misshandlung in einem Klima von Angst, Kontrolle und Isolierung (vgl. Tjaden \& Thoennes 2000a und b) in Sinne von „domestic terrorism“.

Die Forschung zu diesem interessanten aber auch sensiblen Thema muss weiterentwikkelt werden. Bislang weist sie einige Mängel bzw. zumindest kritische Aspekte auf, wie die Synopse von Johnson zeigt: 70\% von 79 Studien verwendeten dasselbe Instrument (Conflict Tactics Scale): methodische Verzerrungen können zu verzerrten Ergebnisse führen. 29 der Studien befragten ausschließlich High School Schüler/innen und College-Student/innen oder dating couples (nicht verheiratet, kein gemeinsamer Haushalt). Das bedeutet, dass die Ergebnisse, die bei dieser Zielgruppe gewonnen wurden, nicht verallgemeinerbar sind. Andere Studien verfolgten andere Fragestellungen oder Zielsetzungen oder es handelte sich um klinische Untersuchungsgruppen (Kimmel 2002). Auch hier ist keine Verallgemeinerung möglich.

Inzwischen hat sich als Standard durchgesetzt, dass bei Befragungen wegen häuslicher Gewalt nicht automatisch ausschließlich von weiblichen Opfern und männlichen Tätern ausgegangen werden kann, sondern dass das Geschlecht nachgefragt wird. Methodische Probleme bei der Erhebung von Gewalt in Partnerschaften sind identifiziert worden und werden zukünftig nach Möglichkeit vermieden. Die repräsentative Studie „Lebenssituation, Sicherheit und Gesundheit von Frauen in Deutschland" (Schrött- 
le:2004) und die Pilotstudie „Gewalt gegen Männer“ (Forschungsgruppe 2004) arbeiteten wie schon die Studie von Tjaden und Thoennes (2000) mit den gleichen Skalen von Gewalthandlungen.

Im Laufe seiner Arbeit stellte Johnson (2005) ein weiteres Muster von Gewalthandeln vor uns nannte es ,violent resistence“. Nicht gemeint ist hier Gegenwehr oder Selbstverteidigung in akuten Gewaltsituationen. Es geht vielmehr um eine gewalttätige Reaktion im Sinne von Vergeltung im Kontext von „intimate terrorism“. Erfasst werden hier vor allem Angriffe von Frauen auf Partner, unter denen sie jahrelang gelitten haben. Wenn bei diesen Taten der Kontext ausgeblendet bleibt, wird übersehen, dass diese Frauen lange Zeit Opfer meist schwerer Gewalt waren, bevor sie selbst zugeschlagen haben. Diese Erkenntnisse sind wichtig für die Intervention in akuten Situationen. US-amerikanische Forschung zeigt, dass eine wachsende Anzahl von Frauen gleichzeitig mit den Männern von der Polizei in Gewahrsam genommen oder der Wohnung verwiesen werden, weil sie ebenfalls gewalttätig waren. Wenn Angriff und Verteidigung nicht unterschieden werden, weil den Einsatzkräften die Kriterien dafür fehlen, wird schützende Intervention ungerecht. Gleiches gilt dafür, wenn eindimensional davon ausgegangen wird, dass Männer immer Täter und Frauen immer Opfer sind. Es braucht Wissen und Kompetenzen zur Differenzierung.

Die geschlechtstypisch verzerrte Sichtweise auf Frauen als Opfer und Männer als Täter ist kein Frauenproblem. Geschlechtsunterschiede bei der moralischen Beurteilung von Gewalt in Paarbeziehungen finden sich ebenso bei Männern (Gabriel, Gassmann, Locher 2007). Die gesellschaftlichen Konstruktionen von Männlichkeit lassen eine realistische Wahrnehmung der Problematik nicht zu. Männer distanzieren sich sowohl von männlichen Opfern als auch von männlichen Tätern. Sie erachten das Gewalthandeln von Frauen als weniger schwerwiegend und schreiben es eher situativen Faktoren zu. Dies entspricht allerdings dem Stand der Forschung zu „situative couple violence“. Ein traditionelles Geschlechterbild wird allerdings dann sichtbar, wenn männliche Täter stärker für ihr Verhalten verantwortlich gemacht und als unsympathischer gesehen werden als Täterinnen. Von Tätern nehmen Männer eher an, dass sie Verletzungen beabsichtigen als von Täterinnen. Die moralische Bewertung von Gewalt bei beiden Geschlechtern durch Männer bestätigt somit einerseits vorliegende Erkenntnisse, andererseits zeigt sie bestehende Geschlechterklischees auf.

Wenn von Gewalt von Frauen in Partnerschaften gesprochen wird, wird in der Regel an Gewalt gegen Männer gedacht. Gewalt in lesbischen Beziehungen blieb ein marginaler Aspekt am Rande der Diskussion. Aber es gibt durchaus neue Forschung dazu (Hester, Donovan, Holmes, McCarry 2006, Ohms 1993, Giorgio u.a. 2007). $20 \%$ bis $30 \%$ der befragten Frauen berichten von häuslicher Gewalt durch Partnerinnen im Laufe ihres Erwachsenenlebens. Dies ist eine klare Parallele zu Gewalt in heterosexuellen Partnerschaften (25\%, Schröttle u.a. 2004). Folgen und Erleben der Gewalt durch die Partnerin weisen kaum Unterschiede zu Gewalt durch männliche Beziehungspartner auf. Auch in gleichgeschlechtlichen Partnerschaften ist die Gewalt von Frauen deutlich weniger verletzungsträchtig und deutlich seltener sexuelle Gewalt. Die Frauen, die von sich selbst sagen, sie seien „misshandelt“ worden, haben in einem Muster von „intimate terrorism" gelebt.

\section{Welche Fragen können Diskussion und Forschung weiterbringen?}

Wie kann Gewalt durch Frauen in Partnerschaften stärker diskutiert werden, ohne ständig Kurzschlüsse zu fördern? Es gilt die Schwierigkeiten beim identifizieren von Gewalttätigen und Gewalterleidenden in all ihrer Komplexität und Widersprüchlichkeit offensiv zum Thema zu machen. Nur so kann vermieden werden, dass Unsicherheiten und Irrtümer in der Interventionspraxis zu Ungerechtigkeiten führen, wie es Forschung aus den USA bereits belegt.

Welche Angebote gibt es für Täterinnen? Sind sie geeignet? Es gilt, bestehende Angebot für männliche Täter daraufhin zu prüfen, welche Elemente auf die Situation gewalttätiger Frauen und Mädchen übertragbar sind und welche neu und spezifisch entwickelt werden müssen (vgl. Henning u.a. 2003). Erkenntnisse neuer Forschung, dass junge Frauen Gewalt einsetzen und dies nicht als Nachahmung männlichen Verhaltens, sondern als weiblich im Sinne einer wehrhaften Weiblichkeit verstehen, geben hier zu denken (Silkenbeumer 2007, Bruhns 2006). Es muss geprüft werden, inwieweit diese Ergebnisse auf die Gewalt von Frauen in Partnerschaften übertragbar sind oder ob es sich um gänzlich unterschiedliche Phänomene handelt. Zukünftig sollte als Orientierung das Konzept einer durchset- zungsfähigen aber gewaltfreien Weiblichkeit diskutiert werden.

Gewalt von Männern gegen Männer sollte in ihrer destruktiven gesellschaftsschädigenden Wirkung ernst und wichtig genommen werden und die Gewalt von Frauen gegen Männer im Rahmen ihrer empirischen Realität jenseits von ideologischen Instrumentalisierungen diskutiert werden.

\section{Literatur:}

Archer, J. (2000) Sex differences in aggression between heterosexual partners: A meta-analytic review, in: Psychological Bulletin, 126 (5), pp 651-680

Artz, Siblle (1998) Sex, Power, \& the Violent School Girl, Toronto, Trifolium Books

Bruhns, Kirsten/Wittmann, Svendy (2006) Umstände und Hintergründe der Einstellungen von Mädchen zur Gewalt. Fachwissenschaftliche Analyse. In: Wilhelm Heitmeyer/Monika Schröttle (Hrsg.): Gewalt. Beschreibungen - Analysen - Prävention. Schriftenreihe der Bundeszentrale für politische Bildung, Band 563. Bonn, S. 294-317

Forschungsgruppe Gewalt gegen Männer (2004) Gewalt gegen Männer - Personale Gewaltwiderfahrnisse von Männern in Deutschland. Ergebnisse der Pilotstudie, www.bmfsfj.de/ Forschungsnetz/Forschungsberichte

Gabriel, Ute; Gassmann, Sonya; Locher, Ruth (2007) Geschlechtsunterschiede bei der moralischen Beurteilung häuslicher Gewalt, Trauma und Gewalt, 1. Jahrgang, Heft 2 Mai 2007, Klett-Cotta, Stuttgart, S. 42-52

Giorgio, Grace (2002), Speaking Silence: Definitional Dialogues in Abusive Lesbian Relationships, Violence against Women, Jg. 8, H. 10, S. 1233-1259.

Gloor Daniela und Meier Hanna (2003): Gewaltbetroffene Männer - wissenschaftliche und gesellschaftlich-politische Einblicke in eine Debatte. In: FamPra.ch, die Praxis des Familienrechts Nr. 3 2003, S. 526-547.

Helfferich, Cornelia/Lehmann, Katrin/Kavemann, Barbara/Rabe, Heike (2004), Wissenschaftliche Untersuchung zur Situation von Frauen und zum Beratungsbedarf nach einem Platzverweis bei häuslicher Gewalt, Sozialministerium Baden-Württemberg (Hg.), Stuttgart.

Henning, Kris; Jones, Angela; Holdford, Robert (2003) Treatment Needs of Women Arrested for Domestic Violence. A Comparison With Male Offenders, Journal of interpersonal violence, August 2003

Hester, Marianne; Donovan, Catherine; Holmes, Jonathan; McCarry, Melanie (2006) Comparing Domestic Abuse in Same Sex and Heterosexual Relationships, Initial report from a study funded by the Economic \& Social Research Council, Award No. RES-000-230650

Johnson, Michael P. (1995) Patriarchal terrorism and common couple violence: Two forms of violence against women, Journal of Marriage and the Family, 57, S. 283-294.

Johnson, MP; Leone, JM. The differential effects on intimate terrorism and situational couple issues. Journal of Family Issues. 2005;26:322-349.

Kavemann, Barbara (1995) Das bringt mein Weltbild durcheinander - Frauen als Täterinnen in der feministischen Diskussion sexueller Gewalt in: Elliott, M: Täterinnen - Sexueller Missbrauch an Mädchen und Jungen, Ruhnmark, S. 13-41 
Kavemann, Barbara (1998) Frauen als Täterinnen - Frauen, die Mädchen und Jungen sexuell missbrauchen, in: Wodtke-Werner, Verena (Hg.) Nicht wegschauen - Vom Umgang mit Sexualstraftätern, Weinheim, S. 31-44

Kavemann, Barbara (2003): Zur Debatte um Männer als Opfer und Frauen als Täterinnen häuslicher Gewalt. In: Frauenhauskoordinierung e.V. (Hrsg.): Sonderinfo 6 zum Aktionsplan der Bundesregierung zur Bekämpfung von Gewalt gegen Frauen. Frankfurt/M., S. 52-55.

Kavemann, Barbara (1997) Zwischen Politik und Professionalität: Das Konzept der Parteilichkeit, in: Hagemann-White, Carol; Kavemann, Barbara; Ohl, Dagmar: Parteilichkeit und Solidarität, Bielefeld, S. $179 \mathrm{ff}$

Kimmel, Michael S. (2002) "Gender Symmetry" in Domestic Violence. A substantive and methodological research review, Violence against Women Vol.8, Nr. 11 / 2002, pp 1332 $-1363$
Lenz, Hans-Joachim (Hg) (2000): Männliche Opfererfahrungen. Problemlagen und Hilfeansätze in der Männerberatung, Weinheim

Ohms, Constanze (1993) Mehr als das Herz gebrochen - Gewalt in lesbischen Beziehungen, Berlin

Piispa, Minna / Statistiks Finnland (2002) Complexity of Patterns of Violence Against Women in heterosexual Partnership, in: Violence against women Vol. 8 No. 4, pp. 873-900

Schröttle, Monika, Müller, Ursula; Glammeier, Sandra (2004) Lebenssituation, Sicherheit und Gesundheit von Frauen in Deutschland, BMFSFJ (Hg.) abzurufen unter www.bmfsfj. de/Forschungsnetz/Forschungsberichte

Silkenbeumer, Mirja (2007): Biografische Selbstentwürfe und Weiblichkeitskonzepte aggressiver Mädchen und junger Frauen. Münster, Lit Verlag.

Thürmer-Rohr, Christina (1989), Frauen in Gewaltverhältnissen, in: Studienschwerpunkt Frauenforschung TU-Berlin (Hg.)Mittäterschaft und Entdeckungslust, Berlin.
Tjaden, Patricia; Thoennes, Nancy (2000 a) Full Report of the Prevalence, Incidence and Consequences of Violence Against Women, National Institute of Justice, NCJ 183781, US Department of Justice

Tjaden, Patricia; Thoennes, Nancy (2000 b) Prevalence and consequences of male-to-female and female-to-male partner violence as measured by the National Violence Against Women Survey, Violence Against Women 6/2000, pp118-141

Die Autorin: Prof. Dr. Barbara Kavemann, Dipl. Soziologin, lehrt an der Katholischen Hochschule für Sozialwesen, Berlin und arbeitet seit 1978 zu Fragen der Gewalt im Geschlechterverhältnis und der sexualisierten Gewalt gegen Kinder und Jugendliche, vorwiegend in der Praxisevaluation und der Fortbildung.

\section{Inhaftierte Frauen - eine aktuelle Bestandsaufnahme des Frauenstrafvollzugs in Deutschland}

Ina Funk

\begin{abstract}
Möchte man sich im Rahmen einer wissenschaftlichen Arbeit mit dem Thema Frauenstrafvollzug befassen, so stößt man bereits bei der Suche nach geeigneter soziologischer und kriminologischer Fachliteratur an Grenzen. Nicht nur in quantitativer, sondern auch in qualitativer Hinsicht werden Missstände deutlich, denn es gibt kaum aktuelle Bestandsaufnahmen (Maelicke 1995, BAGS e.V. 1998, Zolondek 2007) zur Situation von weiblichen Inhaftierten. Dass Frauen als Bestrafte nur marginal in der Öffentlichkeit wahrgenommen werden, kann sowohl durch die geringe Medienpräsenz als auch durch deren schwache gesellschaftliche und sozialpolitische Beachtung bestätigt werden. Als eine mögliche Erklärung für das mangelnde öffentliche Interesse könnte sich in diesem Zusammenhang die niedrige Beteiligung der Frauen an der Gesamtkriminalität erweisen. Denn von den insgesamt am 31.03.2007 im Strafvollzug untergebrachten 75.719 Gefangenen waren lediglich 5,1 \% weiblichen Geschlechts. (vgl. Strafvollzugsstatistik 2007 a, S. 5) Hat die geringe Anzahl weiblicher Gefangener Auswirkungen auf den Frauenvollzug? Ist frauenspezifischer Vollzug überhaupt möglich?
\end{abstract}

Im Zuge einer wissenschaftlichen Arbeit beschäftigte ich mich 2007/2008 mit diesen Fragen und führte hierzu eine Untersuchung zur aktuellen Situation des Frauenstrafvollzugs in der Bundesrepublik durch. Die folgenden Ausführungen basieren auf den hieraus gewonnenen Erkenntnissen, die ich mit Hilfe einer Befragung der Justizministerien und Haftanstalten der einzelnen Bundesländer sowie der Auswertung aktueller Statistiken ermitteln konnte.

\section{Unterbringungsformen}

Frauen werden bundesweit in insgesamt 45 Haftanstalten inhaftiert. Bei lediglich sechs dieser Anstalten handelt es sich um eigenständige Frauenvollzugsanstalten. Diese befinden sich in den Bundesländern BadenWürttemberg (JVA Schwäbisch Gmünd), Bayern (JVA Aichach), Berlin (JVA für Frauen Berlin), Hessen (JVA Frankfurt am Main III), Niedersachsen (JVA für Frauen Vechta) und Nordrhein-Westfalen (JVA Willich II).

Am 31.03.2007 wurden dort 1.927 Frauen untergebracht. In Relation zu den insgesamt im Frauenstrafvollzug inhaftierten 4.068 weiblichen Gefangenen befanden sich dem- nach $47,4 \%$ in besonderen Frauenvollzugseinrichtungen.

Die zentrale Unterbringung in einer der wenigen selbständigen Haftanstalten bedeutet für viele Frauen, weit entfernt von ihrem Heimatort und damit weit weg von ihren Freunden und vor allem von ihrer Familie, ihren Ehemännern und Kindern leben zu müssen. Um den Kontakt aufrechtzuerhalten, sind Angehörige gezwungen, lange Anfahrtswege auf sich zu nehmen. Die damit verbunden Kosten können von vielen nicht oder nur selten getragen werden. (vgl. Kawamura-Reindl 2009, S. 356; Obermöller 1999, S. 135; Stöckle-Nicklas 1989, S. 113 f.; Zolondek 2007, S. 60) Dies kann langfristig zur Folge haben, dass soziale Beziehungen nach außen - vor allem bei Gefangenen, die eine lange Haftstrafe zu verbüßen haben - abbrechen und Frauen nach der Haft auf sich alleine gestellt sind. Die Integration in das „Leben in Freiheit" wird damit erheblich erschwert.

Auch können Vollzugslockerungen, die ebenfalls der Erhaltung von externen Kontakten und einer optimalen Entlassungsvorbereitung dienen sollen, nicht wahrgenommen werden. Denn selbst wenn sie hinsichtlich 